\title{
NUEVO NOMBRE PARA COPÉPODO DESCRITO \\ EN EL ARCHIPIÉLAGO CUBANO \\ (COPEPODA: SIPHONOSTOMATOIDA: ASTEROCHERIDAE)
}

\begin{abstract}
Carlos Varela
Department of Marine Biology and Fisheries, Rosenstiel School of Marine and Atmospheric Science, University of Miami. varela06@gmail.com
\end{abstract}

\section{RESUMEN}

Se propone un nuevo nombre, para eliminar la homonimia, en una especie del género Asterocheres Boeck, 1859 descrita para el archipiélago cubano.

Palabras claves: Copepoda, Siphonostomatoida, Asterocheres, homonimia.

\section{ABSTRACT}

A new name is proposed for homonymy elimination in Asterocheres Boeck, 1859, species described from Cuban archipelago.

Key words: Copepoda, Siphonostomatoida, Asterocheres, homonymy.

El género Asterocheres Boeck, 1859 es el que mayor número de especies presenta dentro de la familia Asterocheridae Giesbrecht, 1899. De este género se han registrado hasta el presente nueve especies para el archipiélago cubano. En Varela (2012) se describen tres nuevas especies del género Asterocheres. Por una lamentable coincidencia una de las especies fue nombrada como otra previamente descrita por Nair y Pillai (1984), para dar solución a esta homonimia y de acuerdo con el Código Internacional de Nomenclatura Zoológica (1999), se propone el cambio detallado a continuación.

\section{Sistemática}

Asterocheres lalanai nuevo nombre

Asterocheres stocki Varela, 2012 (Solenodon 10: 18-22 pp, Figs. 6-7), homónimo de Asterocheres stocki Nair y Pillai, 1984 [Records of the Zoological Survey of India 81 (3/4): 357-362 pp, Figs. 1-19].

Holotipo. Hembra no ovígera. CUBA. Recolectada en el sublitoral adyacente al Acuario Nacional de Cuba, provincia de Ciudad de La Habana el 14.vi.2009. Asociada a la esponja Niphates digitalis. Depositado en ANC 07.2.1.057.

Etimología. Esta especie está dedicada al colega y amigo Dr. Rogelio Lalana Rueda, profesor de varias generaciones de biólogos marinos cubanos. 


\section{LITERATURA CITADA}

International Commission of Zoological Nomenclature. 1999. International Code of Zoological Nomenclature. Fourth Edition. International Trust for Zoological Nomenclature 1999, The Natural History Museum, London.

Nair, B. U. y N. K. Pillai. 1984. On three new species of Asterocherids copepods, with a redescription of Indomyzon quasimi Ummerkutty. Records of the Zoological Survey of India 81 (3/4): 357-372.

Varela, C. 2012. Tres nuevas especies de Asterocheres (Crustacea: Copepoda: Siphonostomatoida), con un nuevo registro para Cuba. Solenodon 10:8-22.

[Recibido: 07 de marzo, 2013. Aceptado para publicación: 06 de mayo, 2013] 